\title{
Quantification of the neurochemical profile using simulated macromolecule resonances at $3 \mathrm{~T}$
}

\author{
Benoît Schaller ${ }^{a}$, Lijing Xin ${ }^{b *}$, Cristina Cudalbu ${ }^{a}$ and Rolf Gruetter ${ }^{a, b, c}$
}

\begin{abstract}
The broad resonances underlying the entire ${ }^{1} \mathrm{H}$ NMR spectrum of the brain, ascribed to macromolecules, can influence metabolite quantification. At the intermediate field strength of $3 \mathrm{~T}$, distinct approaches for the determination of the macromolecule signal, previously used at either 1.5 or $7 \mathrm{~T}$ and higher, may become equivalent. The aim of this study was to evaluate, at $3 \mathrm{~T}$ for healthy subjects using LCModel, the impact on the metabolite quantification of two different macromolecule approaches: (i) experimentally measured macromolecules; and (ii) mathematically estimated macromolecules. Although small, but significant, differences in metabolite quantification (up to $23 \%$ for glutamate) were noted for some metabolites, 10 metabolites were quantified reproducibly with both approaches with a Cramer-Rao lower bound below $\mathbf{2 0} \%$, and the neurochemical profiles were therefore similar. We conclude that the mathematical approximation can provide sufficiently accurate and reproducible estimation of the macromolecule contribution to the ${ }^{1} \mathrm{H}$ spectrum at 3 T. Copyright $\odot 2013$ John Wiley \& Sons, Ltd.
\end{abstract}

Keywords: in vivo ${ }^{1} \mathrm{H}$ MRS; spin echo full intensity acquired localized (SPECIAL); macromolecule; spline baseline; quantification differences

\section{INTRODUCTION}

Localized ${ }^{1} \mathrm{H}$ MRS allows direct and noninvasive insights into brain metabolism. The spectral resolution and signal-to-noise ratio of ${ }^{1} \mathrm{H}$ MRS improve with the main magnetic field $B_{0}(1)$, enabling gains in accuracy and precision of the quantification of many brain metabolites constituting the neurochemical profile at short TE (2-8). However, the accuracy of the metabolite quantification at short TE is hampered by the presence of broad resonances, which underlie the whole ${ }^{1} \mathrm{H}$ spectrum, ascribed to macromolecules (e.g. cytosolic proteins) and characterized by short $T_{1}$ and $T_{2}$ (9-11) and an apparent diffusion coefficient 10-20 times lower than that of metabolites (12). In addition, the macromolecule contribution to the ${ }^{1} \mathrm{H}$ spectrum may change with pathology (13-16). Thus, an accurate and appropriate estimation of the macromolecule contribution to the ${ }^{1} \mathrm{H}$ spectrum is desired to avoid quantification errors of the neurochemical profile (13,17-19).

Several strategies to assess the macromolecule signal have been explored, which are dependent on the field strength (17-26). At low field strength (below 3T), macromolecules have generally been estimated mathematically using spline or wavelets (22), or during a pre-processing step using the early time points of the time domain signal, which are considered to represent mainly the macromolecule signal because of the relatively short $T_{2}{ }^{*}(26)$. However, at high field strength (above $3 \mathrm{~T}$ ), the linewidth of the macromolecule resonances approaches that of the J-coupled metabolite resonances, compromising a mathematical estimation of the macromolecule signal, which cannot be differentiated from the metabolite resonances $(11,27,28)$. Therefore, the in vivo acquisition of the macromolecule contribution is required for an accurate quantification of the neurochemical profile $(17,18,21,29)$.

However, $3 \mathrm{~T}$ is an intermediate field strength for which the macromolecule signal might be reliably estimated either mathematically or experimentally, and the appropriate technique is still under debate (19). A recent study (25) has investigated two approaches to measure the macromolecule signal contribution to the metabolite quantification at $3 \mathrm{~T}$ using QUEST (QUantitation based on quantum ESTimation) (23), and has reported that the metabolitenulling technique results in an increased accuracy of the quantification and a wider range of quantified metabolites relative to the initial signal truncation technique in the time domain. However, the measurement of the macromolecules with the metabolitenulling technique is time consuming.

\footnotetext{
* Correspondence to: L. Xin, Ecole Polytechnique Federale de Lausanne, SBIPMC-LIFMET, Station 6, 1015 Lausanne, Switzerland.

E-mail: lijing.xin@epfl.ch

a B. Schaller, C. Cudalbu, R. Gruetter

Laboratory of Functional and Metabolic Imaging, Ecole Polytechnique Federale de Lausanne, Lausanne, Switzerland

b L. Xin, R. Gruetter

Department of Radiology, University Hospitals of Lausanne, Lausanne, Switzerland

c R. Gruetter

Department of Radiology, University Hospitals of Geneva, Geneva, Switzerland
}

Abbreviations used: AMARES, advanced method for accurate, robust and efficient spectra; ANOVA, analysis of variance; Asp, aspartate; $C r$, creatine; CRLB, Cramér-Rao lower bound; FASTMAP, fast automatic shimming technique by mapping along the projections; GABA, $\gamma$-aminobutyric acid; Glc, glucose; Gln, glutamine; Glu, glutamate; GSH, glutathione; Ins, myo-inositol; Lac, lactate; $N A A, N$-acetylaspartate; NT, number of transients; $P C r$, phosphocreatine; $P E$, phosphorylethanolamine; QUEST, QUantitation based on quantum ESTimation; Scyllo, scyllo-inositol; SD, standard deviation; SPECIAL, spin echo full intensity acquired localized; Tau, taurine; tCho, total choline; TNAA, total NAA; $V A P O R$, variable power RF pulses with optimized relaxation delays. 
As an alternative method, LCModel is widely used and allows accurate metabolite quantification in the frequency domain $(20,30-32)$. The macromolecule signal is thus approximated by a spline function built into LCModel and, optionally, with simulated macromolecule resonances. The experimental measurement of the macromolecules has not been compared previously with the mathematical estimation investigated with LCModel at $3 \mathrm{~T}$.

Therefore, the aim of this study was to evaluate the differences between the experimentally measured macromolecules and the mathematically estimated macromolecules, and to determine their relative impact on the metabolite quantification at $3 \mathrm{~T}$.

\section{MATERIALS AND METHODS}

\section{Subjects and MR protocol}

Eleven healthy subjects (three women and eight men; age, 20-30 years) gave informed consent according to the procedure approved by the local ethics committee. The experiments were performed on a 90-cm-bore clinical 3-T scanner (Magnetom Trio, a Tim System, Syngo MR VB17 Siemens, Erlangen, Germany) equipped with a whole-body gradient coil [40 $\mathrm{mT} / \mathrm{m}, 200 \mathrm{mT} /(\mathrm{m} \mathrm{ms})]$, and data were acquired using a transverse electromagnetic volume coil (MR Instruments, Inc., Minneapolis, MN, USA). The volume of interest $\left(20 \times 20 \times 25 \mathrm{~mm}^{3}\right)$ was located in the frontal lobe predominantly composed of gray matter and surrounded by a shim volume $\left(30 \times 30 \times 35 \mathrm{~mm}^{3}\right)$ (inset Fig. 1). First- and second-order shims were adjusted using automated FAST (EST) MAP (fast automatic shimming technique by mapping along the projections) $(33,34)$, which provided a homogeneous $B_{0}$ field over the volume of interest.

Macromolecule spectra were acquired with the spin echo full intensity acquired localized (SPECIAL) sequence [TR/TE $=4000 / 6 \mathrm{~ms}$; vector size, 2048 pts; number of transients $(\mathrm{NT}), 32 \times 4](4,35)$ preceded by an inversion pulse (duration, $5.12 \mathrm{~ms}$; bandwidth, $4 \mathrm{kHz}$; peak $B_{1}$ amplitude $\gamma B_{1 \mathrm{max}} / 2$ of $1.4 \mathrm{kHz}$ ) to minimize the metabolite contributions. A VAPOR (variable power RF pulses with optimized relaxation delays) water suppression interleaved with blocks of outer volume suppression (36) was placed prior to the sequence to minimize the water signal and lipid signal from extracerebral fat tissue. The location of the voxel and the placement of the outer volume suppression bands were carefully positioned to minimize lipid contamination. A preliminary experiment was conducted with a healthy subject to measure the macromolecule signal with a set of different inversion times ( $\mathrm{TI}=650,700,750$ and $800 \mathrm{~ms}$ ) to determine the optimum $\mathrm{TI}$ for the acquisition of the macromolecule spectrum with minimum metabolite residuals. Then, the macromolecule signal was acquired with a fixed TI of $750 \mathrm{~ms}$ for four subjects (four men). In vivo ${ }^{1} \mathrm{H}$ spectra were acquired for seven subjects (three women and four men) using the SPECIAL localization sequence (TR/TE $=4000 / 6$ ms; vector size, 2048 pts; NT = 148) without an inversion pulse.

\section{Data processing}

After Fourier transformation, individual spectra were manually phase corrected. Then, a frequency correction was applied to all spectra for the small $B_{0}$ drift occurring during the acquisition by aligning the macromolecule peak at 0.89 ppm using Matlab, as described in Tkac and Gruetter (36). Finally, spectra were averaged over four subjects in a summed macromolecule spectrum of 512 scans (128 scans per subject). As a result of

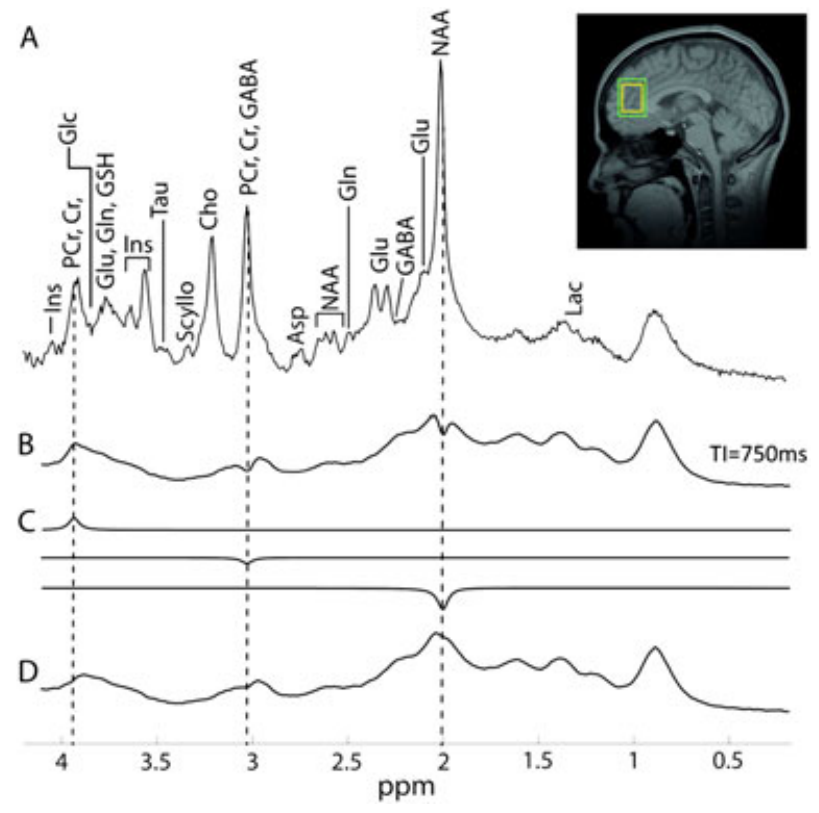

Figure 1. (A) ${ }^{1} \mathrm{H}$ spectrum acquired with SPECIAL $[\mathrm{TR} / \mathrm{TE}=4000 / 6 \mathrm{~ms}$; number of transients (NT), 148] allowing the quantification of 14 metabolites with Cramér-Rao lower bound (CRLB) $<30 \%$. Asp, aspartate; $\mathrm{Cr}$, creatine; GABA, $\gamma$-aminobutyric acid; Glc, glucose; Gln, glutamine; Glu, glutamate; GSH, glutathione; Ins, myo-inositol; Lac, lactate; NAA, Nacetylaspartate; PCr, phosphocreatine; Scyllo, scyllo-inositol; Tau, taurine. (B) The macromolecule spectrum measured with the inversion recovery technique (NT $=512, \mathrm{TI}=750 \mathrm{~ms}$ ) before correction averaged over four subjects. Metabolite residuals were observed for $\mathrm{Cr}$ (3.01 and $3.9 \mathrm{ppm}$ ) and NAA (2.01 ppm) and were fitted with jMRUI (AMARES) (C). (C) was subtracted from (B) to provide a metabolite-free macromolecule spectrum (D). Inset: location of the voxel $\left(20 \times 20 \times 25 \mathrm{~mm}^{3}\right)$ surrounded by the shim volume $\left(30 \times 30 \times 35 \mathrm{~mm}^{3}\right)$ in the frontal cortex. Spectra were line broadened by $2 \mathrm{~Hz}$.

the heterogeneity of the $T_{1}$ value of the metabolites (27), the averaged macromolecule spectrum contained small metabolite residuals.

First, the water residual was filtered from the macromolecule spectrum and metabolite residuals were modeled with jMRUI using AMARES (advanced method for accurate, robust and efficient spectra) (37). As described by Craveiro et al. (38), a set of singlet lorentzian functions was used with constraints on linewidth, frequency, phase and amplitude to compensate for the metabolite residuals that were to be removed. The setting of the parameters included prior knowledge on the phase $\left(0^{\circ}\right.$ for positive residuals and $180^{\circ}$ for negative residuals) and the central frequencies of the metabolite residuals (creatine $(\mathrm{Cr})$ at 3.01 and $3.9 \mathrm{ppm}$ and $\mathrm{N}$-acetylaspartate (NAA) at $2.01 \mathrm{ppm}$ ). The amplitude and linewidth of the singlet lorentzian functions were adjusted in order to fit the metabolite residuals of the macromolecule spectrum. Finally, the modeled fits of the metabolite residuals were subtracted from the macromolecule spectrum to provide a metabolite-free macromolecule spectrum. To reduce the noise of the corrected macromolecule spectrum, a line broadening of $2 \mathrm{~Hz}$ was applied prior the creation of the basis sets.

Plots of the macromolecule spectrum for both approaches and the spline baseline after LCModel analysis are represented in the figures as the mean (thick line) and the standard deviation (SD) ( $n=7$, shaded area). 


\section{Metabolite quantification}

All spectra were fitted and quantified with LCModel (20) using two basis sets. Both included 20 simulated spectra of metabolites using published values for chemical shift and $J$ coupling $(39,40)$, but with different macromolecule approaches: (i) the metabolite-nulled macromolecule spectrum measured with the inversion recovery technique was integrated as prior knowledge in the basis set; and (ii) the mathematically estimated macromolecules using LCModel-simulated macromolecule resonances. Both approaches were completed with built-in spline functions. In the second approach, eight simulated macromolecule resonances provided by LCModel were included in the analysis at the following positions: 0.91, 1.21, 1.43, 1.67, 1.95, 2.08, 2.25 and 3 ppm. Residual water was removed with jMRUI (AMARES) from all the ${ }^{1} \mathrm{H}$ spectra. All ${ }^{1} \mathrm{H}$ spectra were fitted and metabolite concentrations were quantified with LCModel using the two aforementioned basis sets. The LCModel analysis was carried out from 0.2 to $4.2 \mathrm{ppm}$ and a $\mathrm{Cr}$ concentration of $8.5 \mu \mathrm{mol} / \mathrm{g}$ was assumed for the scaling of the metabolite concentrations. The Cramér-Rao lower bound (CRLB) measures the reliability of the fitted parameters by assuming that the model function is correct, and was thus used as a marker of the precision of the quantification $(20,41,42)$. Only fitted metabolite concentration parameters with a mean CRLB below $30 \%$ were retained for further analysis. If a metabolite was quantified with CRLB above $30 \%$ for at least two subjects, this metabolite was excluded from further analysis. However, some metabolites with CRLB above 30\% are reported in Table 1 for comparison. To evaluate the influence of each approach on the quantification, a statistical analysis was performed using a two-way analysis of variance (ANOVA). Metabolite quantification differences with $p$ values below 0.05 were considered to be statistically significant. Metabolite concentration and CRLB are expressed as the mean $\pm S D$.

\section{RESULTS}

\section{Experimentally measured macromolecule signal}

Metabolite residuals were observed in all macromolecule spectra, but were not identical because of the different $T_{1}$ values of the metabolite resonances (27). The identification of the metabolite residuals was based on the time courses of their respective intensities for different Tls and was in agreement with the $T_{1}$ values reported at $3 \mathrm{~T}$ (27). Consequently, a macromolecule spectrum with nulled metabolite resonances could not be acquired. Nevertheless, $\mathrm{TI}=750 \mathrm{~ms}$ was set for the acquisition of the macromolecule spectrum because of the presence of the smallest metabolite residuals, and retained for the following macromolecule acquisitions. These incomplete nulled metabolites were observed in the acquired macromolecule spectrum (Fig. 1B) and consisted of two inverted peaks assigned to NAA (2.01 ppm) and $\mathrm{Cr}$ (3.01 ppm) and a positive peak of $\mathrm{Cr}$ (3.9 ppm).

Next, a macromolecule signal was measured over four subjects and averaged (Fig. 1B). The observed SD varied with the spectral region and was, on average, $3 \pm 1$ from 0.5 to $1.7 \mathrm{ppm}$, $3 \pm 2$ from 1.7 to $3.2 \mathrm{ppm}$ and $7 \pm 4$ from 3.2 to $4.2 \mathrm{ppm}(n=4)$. These values are expressed in arbitrary units (a.u.) and are reported here only as a point of comparison (with the same scale, the macromolecule peak at 0.89 ppm was 100 a.u.).

To eliminate the metabolite residuals of the measured macromolecule spectrum (Fig. 1B), a correction was applied after averaging using jMRUI (AMARES), as described previously (see Data processing and Fig. 1C). It thus provided a metabolite-nulled

Table 1. Cramér-Rao lower bounds (CRLBs) (mean \pm standard deviation) obtained from LCModel analysis of ${ }^{1} \mathrm{H}$ spectra $(n=7)$ using the experimentally measured macromolecules or mathematically estimated macromolecules. Fourteen metabolites were quantified with a mean CRLB below $30 \%$ for both approaches

\begin{tabular}{|c|c|c|}
\hline \multirow[b]{2}{*}{ Metabolite } & \multicolumn{2}{|c|}{ CRLB (\%) } \\
\hline & Experimentally measured macromolecules & Mathematically estimated macromolecules \\
\hline Aspartate & $13 \pm 3$ & $14 \pm 4$ \\
\hline Creatine & $5 \pm 1$ & $5 \pm 1$ \\
\hline Phosphocreatine & $7 \pm 1$ & $7 \pm 1$ \\
\hline$\gamma$-Aminobutyric acid ${ }^{a}$ & $35 \pm 11$ & $17 \pm 3$ \\
\hline Glutamine & $16 \pm 4$ & $28 \pm 10$ \\
\hline Glutamate & $4 \pm 1$ & $6 \pm 1$ \\
\hline Glutathione & $12 \pm 4$ & $10 \pm 3$ \\
\hline myo-Inositol & $5 \pm 1$ & $5 \pm 1$ \\
\hline Lactate & $19 \pm 3$ & $29 \pm 6$ \\
\hline$N$-Acetylaspartate & $2 \pm 0$ & $2 \pm 0$ \\
\hline scyllo-Inositol & $17 \pm 5$ & $18 \pm 6$ \\
\hline Taurine & $26 \pm 10$ & $29 \pm 11$ \\
\hline Glucose & $26 \pm 15$ & $20 \pm 9$ \\
\hline Phosphorylethanolamine & $8 \pm 1$ & $9 \pm 1$ \\
\hline Total NAA & $2 \pm 0$ & $2 \pm 0$ \\
\hline Total choline & $3 \pm 1$ & $3 \pm 1$ \\
\hline Glu + Gln & $4 \pm 1$ & $6 \pm 1$ \\
\hline
\end{tabular}


macromolecule spectrum (Fig. 1D), which formed the basis of the macromolecule model used for the subsequent LCModel analysis.

\section{Macromolecule signal and LCModel analysis}

In vivo ${ }^{1} \mathrm{H}$ NMR spectra were acquired for seven subjects (NT $=148$ ) and were judged to be of high quality based on the high sensitivity of the ${ }^{1} \mathrm{H}$ spectra and the absence of extracerebral lipid signals (Fig. 1A). Adjustments of first- and second-order shims resulted in an average water linewidth of $6.3 \pm 0.1 \mathrm{~Hz}$ (mean \pm SD).

To compare the two different macromolecule estimations, in vivo ${ }^{1} \mathrm{H}$ spectra $(n=7)$ were subjected to LCModel analysis using two approaches: (i) the measured and further processed macromolecule spectrum (averaged over four subjects, see above) (Fig. 2A); and (ii) the mathematical estimation of the macromolecule signal (Fig. 2B). The fitted spectra demonstrated a good match with the acquired ${ }^{1} \mathrm{H}$ spectra for both methods, judging from the flat, noisy fitting residual. It should be noted that, from 0.5 to $4.2 \mathrm{ppm}$, the SD of the mean macromolecule signal fit was below $6 \%$ (Fig. 2A, third spectrum from the top).

To further investigate the similarities between the two approaches, the measured macromolecule signal (experimentally measured macromolecules plus spline baseline) was subtracted from the mathematically estimated macromolecule signal (simulated macromolecules plus spline baseline) (Fig. $3 \mathrm{~A}$ ). The two signals showed good agreement (Fig. 3A, top) and the difference signal (Fig. 3A, bottom) overall exhibited a flat residual, except for some minor changes: the macromolecule signal of the first approach was slightly higher at 1.9 and $3.2 \mathrm{ppm}$, and lower at 2.1, 2.4 and $3.8 \mathrm{ppm}$, than the second approach.

To determine the influence of the two approaches on the quantification, the concentration of the metabolites (mean $\pm S D$ ) and their CRLB (mean \pm SD) were calculated from both LCModel analyses for seven subjects and compared (Fig. 3B and Table 1). The analysis of the ${ }^{1} \mathrm{H}$ spectra allowed the quantification of 14 metabolites for both methods, 10 of which had CRLB below $20 \%$ [aspartate (Asp), total choline (tCho), $\mathrm{Cr}$, phosphocreatine ( $\mathrm{PCr}$ ), glutamate (Glu), glutathione (GSH), myo-inositol (Ins), NAA, scylloinositol (Scyllo) and phosphorylethanolamine (PE)] and four of which had CRLB below 30\% [glucose (Glc), glutamine (Gln), lactate (Lac) and taurine (Tau)]. $\gamma$-Aminobutyric acid (GABA) was quantified with CRLB below $30 \%$ only when using the spline approximation. Overall, the metabolite quantification of the two approaches was similar and the metabolite concentration differences were below $15 \%$ for most metabolites. However, Glu and GSH showed significant concentration differences exceeding the aforementioned $15 \%$ level, i.e. $23 \%(p<0.001)$ and $-33 \%(p<0.05)$, respectively. Low concentration metabolites, such as Gln, GABA and Glc, showed relatively high concentration differences (25-100\%) and are reported in Fig. 3B. However, because of their high CRLB differences (absolute change of 8-20\%), they were excluded from the statistical comparison (i.e. metabolite concentrations are shown, but $p$ values were not reported). It should also be noted that, between the first and second approaches, the GABA concentration doubled, but the CRLB halved in percentage.

\section{DISCUSSION}

This study reports that the neurochemical profile of 10 metabolites can be reliably quantified with CRLB below $20 \%$ at $3 \mathrm{~T}$ for healthy subjects when using two different approaches for macromolecule estimation, namely: (i) the experimentally measured in vivo macromolecule spectrum; and (ii) the mathematical estimation of the macromolecule signal. Metabolite concentration differences between the two approaches were within SD for all the metabolites and below 15\%, except for Glu $(23 \%, p<0.001)$ and

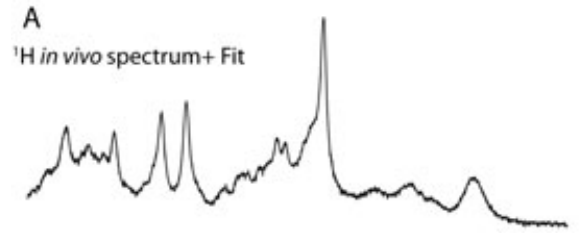

Fitting residual

Measured macromolecules

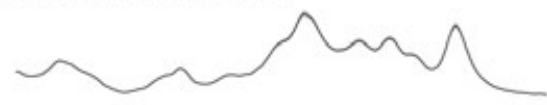

Spline

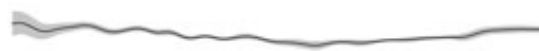

Measured Macromolecules + Spline

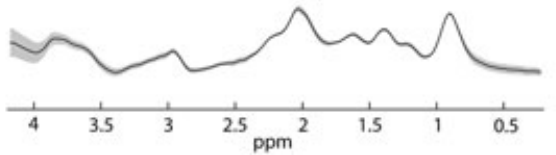

B

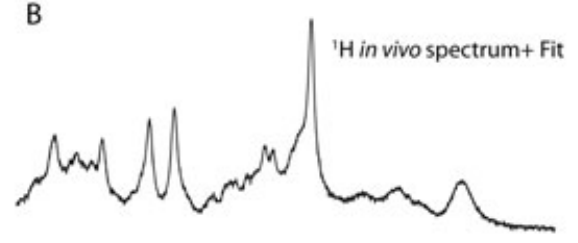

Fitting residual

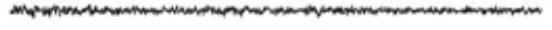

Simulated Macromolecules

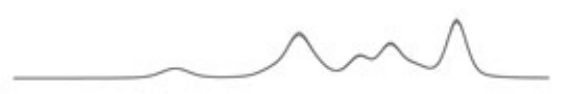

Spline

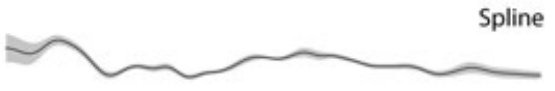

Simulated Macromolecules + Spline

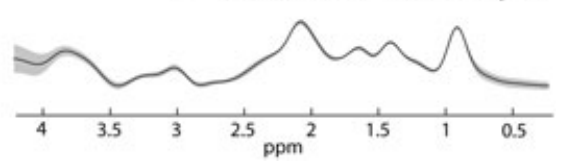

Figure 2. ${ }^{1} \mathrm{H}$ NMR spectra (top; $\mathrm{NT}=148$ ) of seven subjects were quantified with LCModel using the experimentally measured macromolecules (A) or mathematically estimated macromolecules (B). From top to bottom: the ${ }^{1} \mathrm{H}$ spectrum (fitted and real spectrum), fitting residual, corrected macromolecule signal (left) and simulated macromolecules (right), the spline baseline and the sum of the last two. The curves are represented as the mean (black) \pm standard deviation (shaded area). 


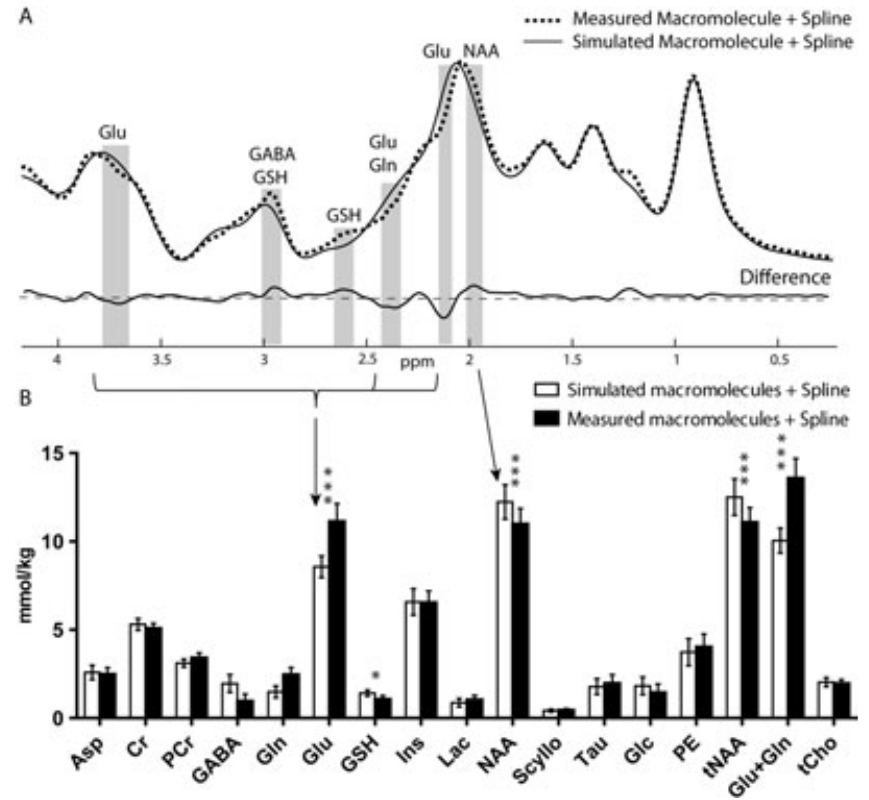

Figure 3. (A) Overlap of the fits obtained after LCModel analysis of the in vivo measured macromolecule spectrum after correction (broken line) and the simulated macromolecules (full line) with their respective spline baseline. Difference signal by subtraction of the two fits was also obtained (bottom). Variations between the fits leading to metabolite quantification differences are highlighted in gray. (B) Metabolite quantification (mean $\pm \mathrm{SD}, n=7$ ) of the ${ }^{1} \mathrm{H}$ spectrum using the mathematically estimated macromolecules (white) and the in vivo measured macromolecules (black). A two-way analysis of variance (ANOVA) measured the significance of the changes between the two approaches $\left({ }^{*} p<0.05\right.$, ${ }^{* * *} p<0.001$ ). Metabolite concentrations were scaled to a creatine concentration of $8.5 \mu \mathrm{mol} / \mathrm{g}$.

GSH $(-33 \%, p<0.05)$. The small disparities of the obtained metabolite concentrations and CRLBs between the two methods were consistent with the differences between the macromolecule estimation of the two approaches.

The macromolecule spectra were experimentally measured by an inversion recovery experiment with an inversion time set to $750 \mathrm{~ms}$ and averaged over four subjects (Fig. 1B). The residual water signal of the averaged macromolecule spectrum was filtered with jMRUI (AMARES) and phase corrected. As a result of the high signal-to-noise ratio, metabolite residuals were identified and removed (Fig. 1C) using jMRUI (AMARES), providing a metabolite-free macromolecule spectrum (Fig. 1D). The small SD of the averaged macromolecules illustrated the overall stability and reproducibility of the experiment, and enabled the use of an averaged measured macromolecule signal (NT $=512$ scans) to quantify the ${ }^{1} \mathrm{H}$ spectra of healthy subjects ( $n=7$ Fig. 2 ). However, the mean SD of the averaged macromolecule spectrum was two-fold higher from 3.2 to $4.2 \mathrm{ppm}$ relative to 0.5 to $1.7 \mathrm{ppm}$ (data not shown). The filtering of the residual water of the macromolecule spectra helped to reduce the SD, but some small variation remained, probably as a result of intersubject variation. In general, the measured macromolecule baseline was extremely stable between subjects, and such stability may be harder to achieve in pathology. Thus, the acquired and processed macromolecule spectrum was used in the LCModel basis set for ${ }^{1} \mathrm{H}$ spectra quantification $(n=7)$.

In vivo ${ }^{1} \mathrm{H}$ spectra were acquired for seven subjects and fitted with LCModel using the same metabolite basis set, but two different macromolecule approaches: the measured macromolecule spectrum or the simulated macromolecule resonances and the built-in spline function. In the first approach (Fig. 2A), the macromolecule fit showed small SD (within 6\%), which probably reflects the small variability of macromolecules between subjects in the frontal lobe. Residual water filtering contributed to the aforementioned small SD and also to a flat spline baseline. Concerning the mathematical approach (Fig. 2B), the simulated macromolecule resonances also exhibited a small SD (within 7\%), but ranged only from 0.91 to $3 \mathrm{ppm}$. Hence, the spline baseline accommodated the entire macromolecule signal in the 3-4-ppm range and showed higher fluctuation in this range.

The concentrations of 13 metabolites were quantified with a mean CRLB below 30\%. The aforementioned differences between the fits of each approach (the measured macromolecules and the mathematically estimated macromolecules) led to some relative quantification differences (Fig. 3 and Table 1). The mathematically estimated macromolecules were slightly higher at 2.1, 2.4 and $3.8 \mathrm{ppm}$, which led to an underestimation of the Glu and Gln concentrations when compared with those obtained using the experimentally measured macromolecules. However, GABA, GSH and NAA concentrations were overestimated, probably as a result of the macromolecule differences at 1.9 and $3.2 \mathrm{ppm}$. The concentration and CRLB differences were within SD for all reliably quantified metabolites (CRLB below 30\%), except for Glu (Fig. 3B). These changes were relatively small, but were systematic, and thus statistically significant, for Glu, NAA, total NAA (tNAA) and Glu + Gln $(p<0.05)$. Gln and Glc are low concentration metabolites and their concentration differences were also significant; however, these changes were comparable with their respective CRLBs when expressed in concentration units, and thus are probably below experimental error, when including, for example, inter-individual differences. The CRLB of the metabolites quantified with CRLB $<30 \%$ (Table 1) differed slightly between the two approaches (within $\pm 2 \%$ ), with the exception of Gln (+12\%), Lac (+10\%) and Glc (-6\%).

GABA is a low concentration metabolite and its concentration difference between the two approaches was also significant. The current literature states a GABA concentration around $1 \mu \mathrm{mol} / \mathrm{g}$ or lower (43-45). In our study, when using the experimentally measured macromolecules, the GABA concentration was $1.0 \pm 0.3$ $\mu \mathrm{mol} / \mathrm{g}(\mathrm{CRLB}=35 \pm 11 \%, n=7)$. However, when using the mathematically estimated macromolecules, the GABA concentration was $2.0 \pm 0.3 \mu \mathrm{mol} / \mathrm{g}(\mathrm{CRLB}=17 \pm 3 \%, n=7)$. Our results suggest that the inclusion of an experimentally measured macromolecule spectrum at $3 \mathrm{~T}$ may provide a GABA concentration that is in good agreement with literature values.

In general, an overestimation or underestimation of the macromolecule signal may influence strongly the CRLB of the metabolites with low concentration but, overall, the metabolite concentrations were fairly similar between the two approaches and were in agreement with previous studies (45-47). The location of the voxel in the frontal lobe suggests a composition of predominantly gray matter. Therefore, the Glu concentration seems to be closer to literature values $(45,47)$ when using the measured macromolecule baseline for the LCModel analysis relative to the mathematical approach.

In addition, the macromolecule signal was investigated (data not shown) using only the built-in spline in the LCModel analysis (i.e. simulated macromolecule resonances were not included). The neurochemical profile between the two mathematical 
approaches was similar, but quantification differences were significant for Glu, Gln and GABA and a higher CLRB for Lac was observed. The Glu, Gln and GABA concentrations obtained when using only the built-in spline to estimate the macromolecule signal were between those obtained with the two approaches studied.

A previous study at $3 \mathrm{~T}(25)$ investigated the approximation of the macromolecule signal contribution by focusing on the measured metabolite-nulled macromolecule signal and the macromolecule baseline modeled by Subtract-QUEST (jMRUI). Gottschalk et al. (25) concluded that the measured macromolecules enabled a more accurate quantification and that the use of the baseline computed by Subtract-QUEST would result in a loss of metabolic information. However, in our study, the profile of the macromolecule signal of the two approaches (experimentally measured and mathematically estimated) showed strong similarities (Fig. 3A) with similar quantification. The aforementioned concentration differences were generally small (Fig. 3B) and CRLBs were equivalent, but generally smaller, when using the measured macromolecules (Table 1). It should also be noted that Asp, Scyllo and PE were quantified with CRLB below $20 \%$ using both methods, in addition to the seven quantified metabolites reported previously (25).

The field dependence of the linewidth, imposing the measurement of macromolecules at high field for reliable quantification (18), does not prevail in our study conducted at $3 \mathrm{~T}$, and no distinction, when using the experimentally measured macromolecule signal or the mathematically estimated macromolecules, could be made based on the metabolite concentration and CRLB provided by LCModel analysis.

Macromolecules may vary with pathology, and can be considered as disease markers (13-16). Practically, macromolecule resonances can be altered differently depending on the studied spectral region and the stage of the disease. In these cases, the adaptability of the mathematical estimation probably allows a closer approximation of the macromolecule baseline than does the use of a general prepared macromolecule signal averaged from healthy volunteers, as the macromolecule resonances can be affected differentially by disease.

We conclude that the experimentally measured macromolecule signal and the mathematically estimated macromolecule signal provide a similar neurochemical profile. Given the small concentration differences of the ensuing LCModel quantification, we suggest that, at $3 \mathrm{~T}$, the mathematical approximation is a sufficient estimation of the macromolecule contribution to ${ }^{1} \mathrm{H}$ spectra at $3 \mathrm{~T}$. As a caveat, small, but significant, differences in the quantification may need to be taken into account when comparing metabolite concentrations obtained with these two different approaches, such as for Glu and for GABA, which were more reliably quantified when using the measured macromolecule baseline.

\section{Acknowledgements}

This work was supported by the Centre d'Imagerie Bio-Médicale (CIBM) of the University of Lausanne (UNIL), the Swiss Federal Institute of Technology Lausanne (EPFL), the University of Geneva (UniGe), the Centre Hospitalier Universitaire Vaudois (CHUV), the Hôpitaux Universitaires de Genéve (HUG) and the Leenaards and the Jeantet Foundations and by a Swiss National Science grant to R. Gruetter. We would like to thank Mélanie Craveiro for her help in the processing of the acquired macromolecule signal. The authors declare no conflict of interest.

\section{REFERENCES}

1. Gruetter R, Weisdorf SA, Rajanayagan V, Terpstra M, Merkle H, Truwit CL, Garwood M, Nyberg SL, Ugurbil K. Resolution improvements in in vivo ${ }^{1} \mathrm{H}$ NMR spectra with increased magnetic field strength. J. Magn. Reson. 1998; 135(1): 260-264.

2. Gonen O, Gruber S, Li BS, Mlynarik V, Moser E. Multivoxel 3D proton spectroscopy in the brain at 1.5 versus $3.0 \mathrm{~T}$ : signal-to-noise ratio and resolution comparison. Am. J. Neuroradiol. 2001; 22(9): 1727-1731.

3. Bartha R, Drost DJ, Menon RS, Williamson PC. Comparison of the quantification precision of human short echo time (1) H spectroscopy at 1.5 and 4.0 Tesla. Magn. Reson. Med. 2000; 44(2): 185-192.

4. Mekle R, Mlynarik V, Gambarota G, Hergt M, Krueger G, Gruetter R. MR spectroscopy of the human brain with enhanced signal intensity at ultrashort echo times on a clinical platform at $3 \mathrm{~T}$ and $7 \mathrm{~T}$. Magn. Reson. Med. 2009; 61(6): 1279-1285.

5. Tkac I, Oz G, Adriany G, Ugurbil K, Gruetter R. In vivo ${ }^{1} \mathrm{H}$ NMR spectroscopy of the human brain at high magnetic fields: metabolite quantification at 4T vs. 7T. Magn. Reson. Med. 2009; 62(4): 868-879.

6. Tkac I, Andersen P, Adriany G, Merkle H, Ugurbil K, Gruetter R. In vivo ${ }^{1} \mathrm{H}$ NMR spectroscopy of the human brain at 7 T. Magn. Reson. Med. 2001; 46(3): 451-456.

7. Tkac I, Henry PG, Andersen P, Keene CD, Low WC, Gruetter R. Highly resolved in vivo ${ }^{1} \mathrm{H}$ NMR spectroscopy of the mouse brain at $9.4 \mathrm{~T}$. Magn. Reson. Med. 2004; 52(3): 478-484.

8. Tkac I, Rao R, Georgieff MK, Gruetter R. Developmental and regional changes in the neurochemical profile of the rat brain determined by in vivo ${ }^{1} \mathrm{H}$ NMR spectroscopy. Magn. Reson. Med. 2003; 50(1): 24-32.

9. Behar KL, Ogino T. Characterization of macromolecule resonances in the ${ }^{1}$ H NMR spectrum of rat brain. Magn. Reson. Med. 1993; 30 (1): 38-44.

10. Behar KL, Rothman DL, Spencer DD, Petroff OA. Analysis of macromolecule resonances in ${ }^{1} \mathrm{H}$ NMR spectra of human brain. Magn. Reson. Med. 1994; 32(3): 294-302.

11. Xin L, Schaller B, Mlynarik V, Lu H, Gruetter R. Proton T(1) relaxation times of metabolites in human occipital white and gray matter at 7 T. Magn. Reson. Med. 2012.

12. Kunz N, Cudalbu C, Mlynarik V, Huppi PS, Sizonenko SV, Gruetter R. Diffusion-weighted spectroscopy: a novel approach to determine macromolecule resonances in short-echo time ${ }^{1} \mathrm{H}-\mathrm{MRS}$. Magn. Reson. Med. 2010; 64(4): 939-946.

13. Seeger U, Klose U, Mader I, Grodd W, Nagele T. Parameterized evaluation of macromolecules and lipids in proton MR spectroscopy of brain diseases. Magn. Reson. Med. 2003; 49(1): 19-28.

14. Mader I, Seeger U, Weissert R, Klose U, Naegele T, Melms A, Grodd W. Proton MR spectroscopy with metabolite-nulling reveals elevated macromolecules in acute multiple sclerosis. Brain 2001; 124(Pt 5): 953-961.

15. Graham GD, Hwang JH, Rothman DL, Prichard JW. Spectroscopic assessment of alterations in macromolecule and small-molecule metabolites in human brain after stroke. Stroke 2001; 32(12): 2797-2802.

16. Howe FA, Barton SJ, Cudlip SA, Stubbs M, Saunders DE, Murphy M, Wilkins P, Opstad KS, Doyle VL, McLean MA, Bell BA, Griffiths JR. Metabolic profiles of human brain tumors using quantitative in vivo ${ }^{1} \mathrm{H}$ magnetic resonance spectroscopy. Magn. Reson. Med. 2003; 49(2): 223-232.

17. Cudalbu C, Beuf $\mathrm{O}$, Cavassil S. In vivo short echo time localized ${ }^{1} \mathrm{H}$ MRS of the rat brain at 7T: influence of two strategies of background-accommodation on the metabolite concentration estimation using QUEST. J. VLSI Signal Proc. Syst. 2009; 55(1): 25.

18. Cudalbu C, Mlynárik V, Xin L, Gruetter R. Quantification of in vivo short echo-time proton magnetic resonance spectra at $14.1 \mathrm{~T}$ using two different approaches of modelling the macromolecule spectrum. Meas. Sci. Technol. 2009; 20: 104034 (7pp).

19. Cudalbu C, Mlynarik V, Gruetter R. Handling macromolecule signals in the quantification of the neurochemical profile. J. Alzheimers Dis. 2012; 31(3): S101-S115.

20. Provencher SW. Estimation of metabolite concentrations from localized in vivo proton NMR spectra. Magn. Reson. Med. 1993; 30 (6): 672-679.

21. Pfeuffer J, Tkac I, Provencher SW, Gruetter R. Toward an in vivo neurochemical profile: quantification of 18 metabolites in shortecho-time (1)H NMR spectra of the rat brain. J. Magn. Reson. 1999; 141(1): 104-120. 
22. Soher BJ, Young K, Maudsley AA. Representation of strong baseline contributions in ${ }^{1} \mathrm{H}$ MR spectra. Magn. Reson. Med. 2001; 45(6): 966-972.

23. Ratiney H, Coenradie $Y$, Cavassila S, van Ormondt D, GraveronDemilly $\mathrm{D}$. Time-domain quantitation of ${ }^{1} \mathrm{H}$ short echo-time signals: background accommodation. MAGMA 2004; 16(6): 284-296.

24. Poullet JB, Sima DM, Van Huffel S, Van Hecke P. Frequency-selective quantitation of short-echo time ${ }^{1} \mathrm{H}$ magnetic resonance spectra. J. Magn. Reson. 2007; 186(2): 293-304.

25. Gottschalk M, Lamalle L, Segebarth C. Short-TE localised ${ }^{1} H$ MRS of the human brain at $3 \mathrm{~T}$ : quantification of the metabolite signals using two approaches to account for macromolecular signal contributions. NMR Biomed. 2008; 21(5): 507-517.

26. Ratiney $H$, Sdika $M$, Coenradie $Y$, Cavassila $S$, van Ormondt $D$, Graveron-Demilly D. Time-domain semi-parametric estimation based on a metabolite basis set. NMR Biomed. 2005; 18(1): 1-13.

27. Mlynarik V, Gruber S, Moser E. Proton T (1) and T (2) relaxation times of human brain metabolites at 3 Tesla. NMR Biomed. 2001; 14(5): 325-331.

28. Deelchand DK, Van de Moortele PF, Adriany G, Iltis I, Andersen P, Strupp JP, Vaughan JT, Ugurbil K, Henry PG. In vivo ${ }^{1} \mathrm{H}$ NMR spectroscopy of the human brain at 9.4 T: initial results. J. Magn. Reson. 2010; 206(1): 74-80.

29. Mlynarik V, Cudalbu C, Xin L, Gruetter R. ${ }^{1} H$ NMR spectroscopy of rat brain in vivo at 14.1 Tesla: improvements in quantification of the neurochemical profile. J. Magn. Reson. 2008; 194(2): 163-168.

30. Mandal PK. In vivo proton magnetic resonance spectroscopic signal processing for the absolute quantitation of brain metabolites. Eur. J. Radiol. 2012; 81(4): e653-e664.

31. Kanowski M, Kaufmann J, Braun J, Bernarding J, Tempelmann C. Quantitation of simulated short echo time ${ }^{1} \mathrm{H}$ human brain spectra by LCModel and AMARES. Magn. Reson. Med. 2004; 51(5): 904-912.

32. Provencher SW. Automatic quantitation of localized in vivo ${ }^{1} \mathrm{H}$ spectra with LCModel. NMR Biomed. 2001; 14(4): 260-264.

33. Gruetter R. Automatic, localized in vivo adjustment of all first- and second-order shim coils. Magn. Reson. Med. 1993; 29(6): 804-811.

34. Gruetter R, Tkac I. Field mapping without reference scan using asymmetric echo-planar techniques. Magn. Reson. Med. 2000; 43(2): 319-323.

35. Mlynarik V, Gambarota G, Frenkel H, Gruetter R. Localized shortecho-time proton MR spectroscopy with full signal-intensity acquisition. Magn. Reson. Med. 2006; 56(5): 965-970.
36. Tkac I, Gruetter R. Methodology of H NMR spectroscopy of the human brain at very high magnetic fields. Appl. Magn. Reson. 2005; 29(1): 139-157.

37. Vanhamme L, van den Boogaart A, Van Huffel S. Improved method for accurate and efficient quantification of MRS data with use of prior knowledge. J. Magn. Reson. 1997; 129(1): 35-43.

38. Craveiro M, Cudalbu C, Gruetter R. Regional alterations of the brain macromolecule resonances investigated in the mouse brain using an improved method for the pre-processing of the macromolecular signal. Proceedings of the 20th Annual Meeting ISMRM, Melbourne, Victoria, Australia, 2012; 1748.

39. Govindaraju V, Young K, Maudsley AA. Proton NMR chemical shifts and coupling constants for brain metabolites. NMR Biomed. 2000; 13(3): 129-153.

40. Xin L, Gambarota G, Mlynarik V, Gruetter R. Proton T2 relaxation time of J-coupled cerebral metabolites in rat brain at 9.4 T. NMR Biomed. 2008; 21(4): 396-401.

41. Cavassila S, Deval S, Huegen C, van Ormondt D, Graveron-Demilly D. Cramer-Rao bounds: an evaluation tool for quantitation. NMR Biomed. 2001; 14(4): 278-283.

42. Cavassila S, Deval S, Huegen C, van Ormondt D, Graveron-Demilly D. Cramer-Rao bound expressions for parametric estimation of overlapping peaks: influence of prior knowledge. J. Magn. Reson. 2000; 143(2): 311-320.

43. Rothman DL, Petroff OA, Behar KL, Mattson RH. Localized ${ }^{1} \mathrm{H}$ NMR measurements of gamma-aminobutyric acid in human brain in vivo. Proc. Natl. Acad. Sci. USA 1993; 90(12): 5662-5666.

44. Terpstra M, Ugurbil K, Gruetter R. Direct in vivo measurement of human cerebral GABA concentration using MEGA-editing at 7 Tesla. Magn. Reson. Med. 2002; 47(5): 1009-1012.

45. Emir UE, Auerbach EJ, Moortele PF, Marjanska M, Ugurbil K, Terpstra $\mathrm{M}, \mathrm{Tkac}$ I, Oz G. Regional neurochemical profiles in the human brain measured by (1) H MRS at $7 \mathrm{~T}$ using local $\mathrm{B}(1)$ shimming. NMR Biomed. 2012; 25(1): 152-160.

46. Bogner W, Gruber S, Trattnig S, Chmelik M. High-resolution mapping of human brain metabolites by free induction decay (1) H MRSI at 7 T. NMR Biomed. 2012; 25(6): 873-882.

47. Mangia S, Tkac I, Gruetter R, Van De Moortele PF, Giove F, Maraviglia $B$, Ugurbil K. Sensitivity of single-voxel ${ }^{1} \mathrm{H}-\mathrm{MRS}$ in investigating the metabolism of the activated human visual cortex at 7 T. Magn. Reson. Imaging 2006; 24(4): 343-348. 